\title{
FrSh61 (MEA) VHL33 Peptide
}

National Cancer Institute

\section{Source}

National Cancer Institute. FrSh61 (MEA) VHL33 Peptide. NCI Thesaurus. Code C29065.

A peptide derived from the tumor suppressor protein Von Hippel-Lindau (VHL). This peptide, sequence: MEAGRPRPCCAR, was constructed based on a frameshift mutation of one of the VHL gene products; the more abundant protein VHLP18(MEA) (where MEA stands for three amino acids, Met-Glu-Ala). Vaccination with FrSh61(MEA)VHL33 peptide may stimulate a cytotoxic T-lymphocyte $(C T L)$ response against tumor cells expressing this VHL mutant protein. 